\title{
DISCRETE-TIME SYSTEMS WITH TIME-VARYING TIME DELAY: STABILITY AND STABILIZABILITY
}

\author{
EL-KÉBIR BOUKAS
}

Received 7 December 2005; Accepted 2 January 2006

This paper deals with the class of linear discrete-time systems with varying time delay. The problems of stability and stabilizability for this class of systems are considered. Given an upper bound and a lower bound on the time-varying delay, sufficient conditions for checking the stability of this class of systems are developed. A control design algorithm is also provided. All the results developed in this paper are in the LMI formalism which makes their solvability easier using existing tools. A numerical example is provided to show the effectiveness of the established results.

Copyright (C 2006 El-Kébir Boukas. This is an open access article distributed under the Creative Commons Attribution License, which permits unrestricted use, distribution, and reproduction in any medium, provided the original work is properly cited.

\section{Introduction}

It is well established in the literature that time delay is the cause of performance degradations for dynamical systems. It can even be, in some circumstances, the cause of instability of the system that we would like to control if such time delay is not taken into account during the design phase. Time delay may occur either in continuous-time or discretetime systems and may be constant or time varying.

The control of systems with time delay has been a hot subject of research in the last decades. Stability and stabilizability problems either for the continuous-time and discrete-time deterministic and stochastic systems with time delay have been tackled and interesting results have been reported in the literature. The reported results are divided into delay-independent and delay-dependent conditions. The delay-independent conditions present more conservatism than delay-dependent conditions since they do not depend on the delay in the system.

For deterministic class of linear systems with time delay, we have seen an increasing interest during the last two decades. Many papers, mainly on the class of deterministic continuous-time linear systems with time delay have been published. Stability and stabilizability problems have been studied and interesting results are available in the literature.

Hindawi Publishing Corporation

Mathematical Problems in Engineering

Volume 2006, Article ID 42489, Pages 1-10

DOI 10.1155/MPE/2006/42489 
However, for deterministic class of discrete-time linear systems with constant time delay only few results have been reported in the literature. The main reason for this, we believe, is that this kind of systems can be transformed to an equivalent system without time delay and then we can use the known results on stability and stabilizability. For more information on some of the developed results for this class of systems, we refer the reader to Boukas and Liu [1], Kim and Park [6], Song and Kim [9], Mukaidani et al. [7], Chang et al. [4], Gao et al. [5], and references therein.

The problems of stochastic stability and stochastic stabilizability have also been tackled by many authors and recently we have seen the publication of different results ranging from delay independent to delay dependent for the continuous-time and the discretetime cases. Several situations were considered including constant time delay, time-varying delay and mode-dependent time delay for the continuous-time systems and constant and mode-dependent time delay for discrete-time systems. For more details on this subject we refer the reader to Boukas and Liu [2], Boukas and Liu [1], and the references therein, respectively, for the continuous-time and discrete-time cases. Other results can be found in [8], [3], and the references therein.

To the best of the author knowledge, the mode-dependent time-delay case for the class of discrete-time linear systems with varying time delay has not been fully investigated and this will be the subject of this paper. Among the references that tackled the delaydependent stability and the stabilizability problems for linear systems with varying time delay, we quote those of Mukaidani et al. [7], Chang et al. [4], Gao et al. [5], and the references therein. The goal of this paper is to develop new LMI-based delay-dependent sufficient conditions for stability and stabilizability for linear discrete-time systems with varying time delay in the state.

The paper is organized as follows. In Section 2, the problem is stated and the objectives of the paper are formulated. The problem of stability for the discrete-time linear system with varying time delay is examined and delay-dependent sufficient conditions are developed in Section 3. In Section 4, the stabilizability problem is investigated and delay-dependent conditions are established. In addition, a design algorithm that stabilizes the resulting closed-loop system is provided. A numerical example is given to show the usefulness of the proposed theoretical results in Section 5.

\section{Problem statement}

The dynamics of the system we consider in this paper are assumed to be described by the following difference equation:

$$
x_{k+1}=A x_{k}+A_{d} x_{k-d_{k}}+B u_{k} \quad x_{k}=\phi_{k}, k \in[-\bar{d}, \ldots, 0],
$$

where $x_{k} \in \mathbb{R}^{n}$ is the state at instant $k, u_{k} \in \mathbb{R}^{p}$ is the control input at instant $k$, the matrices $A \in \mathbb{R}^{n \times n}, A_{d} \in \mathbb{R}^{n \times n}$, and $B \in \mathbb{R}^{n \times p}$ are constant matrices and $d_{k}$ is a positive integer representing the time delay of the system that we assume to be time dependent and satisfies the following:

$$
0 \leq \underline{d} \leq d_{k} \leq \bar{d}
$$

where $\underline{d}$ and $\bar{d}$ are known to be positive and finite integers. 
The aim of this paper is to establish the sufficient conditions that guarantee the stability of the class of system (2.1). We will also tackle the stabilizability problem of this class of systems. LMI-based delay-dependent conditions either for stability or stabilization are targeted.

The control law we will use in the paper is given by the following:

$$
u_{k}=K x_{k}
$$

where $K$ is the control gain to be computed.

Notation 2.1. The notation used in this paper is quite standard. $\mathbb{R}^{n}$ and $\mathbb{R}^{n \times m}$ denote, respectively, the set of the $n$ dimensional space of real vectors and the set of all $n \times m$ real matrices. The superscript " $T$ " denotes the transpose and the notation $X \geq Y$ (resp., $X>$ $Y$ ) where $X$ and $Y$ are symmetric matrices, meaning that $X-Y$ is positive semidefinite (resp., positive definite). $P>0$ means that $P$ is symmetric and positive definite. $\square$ is the identity matrix with compatible dimension. We define $\mathbf{x}_{l}(k)=x_{k+l}, k-d_{k} \leq l \leq k$, noted in the sequel by $\mathbf{x}_{k}$.

\section{Stability}

In this section, we present delay-dependent conditions that can be used to check if the system we are considering is stable. The following result gives what conditions to be satisfied to guarantee that the system (2.1) for $u_{k}=$ for $k \geq 0$ is stable.

THEOREM 3.1. For a given set of upper and lower bounds $\bar{d}$ and $\underline{d}$ for the time varying delay $r_{k}$, if there exist symmetric and positive-definite matrices $P_{1}>0, Q>0$ and $R>0$, and matrices $P_{2}$ and $P_{3}$ such that the following LMIs hold:

$$
\begin{gathered}
Q<R \\
{\left[\begin{array}{ccc}
Q+(\bar{d}-\underline{d}) R-P_{1}-A^{\top} P_{2}-P_{2}^{\top} A & -A^{\top} P_{3}+P_{2}^{\top} & -P_{2}^{\top} A_{d} \\
-P_{3}^{\top} A+P_{2} & P_{1}+P_{3}+P_{3}^{\top} & -P_{3}^{\top} A_{d} \\
-A_{d}^{\top} P_{2} & -A_{d}^{\top} P_{3} & -Q
\end{array}\right]<0,}
\end{gathered}
$$

then, system (2.1) is stable.

Proof. To prove our theorem, let us consider the following change of variables:

$$
x_{k+1}=y_{k} \quad 0=-y_{k}+A x_{k}+A_{d} x_{k-d_{k}} .
$$

Define $\tilde{x}_{k}^{\top}=\left[\begin{array}{lll}x_{k} & y_{k} & x_{k-d_{k}}\end{array}\right]^{\top}$, and consider the following Lyapunov-Krasovskii candidate functional:

$$
V\left(\widetilde{\mathbf{x}}_{k}\right)=V_{1}\left(\tilde{\mathbf{x}}_{k}\right)+V_{2}\left(\tilde{\mathbf{x}}_{k}\right)+V_{3}\left(\tilde{\mathbf{x}}_{k}\right)
$$


4 Discrete-time systems with time-varying time delay

with

$$
V_{1}\left(\tilde{\mathbf{x}}_{k}\right)=\tilde{x}_{k}^{\top} E^{\top} P \tilde{x}_{k}, \quad V_{2}\left(\tilde{\mathbf{x}}_{k}\right)=\sum_{l=k-d_{k}}^{k-1} x_{l}^{\top} Q x_{l}, \quad V_{3}\left(\tilde{\mathbf{x}}_{k}\right)=\sum_{l=-\bar{d}+2}^{-\underline{d}+1} \sum_{m=k+l-1}^{k-1} x_{m}^{\top} R x_{m},
$$

where $Q>0$ and $R>0$, and $E$ and $P$ are, respectively, singular and nonsingular matrices with the following forms:

$$
E=\left[\begin{array}{lll}
\square & 0 & 0 \\
0 & 0 & 0 \\
0 & 0 & 0
\end{array}\right], \quad P=\left[\begin{array}{ccc}
P_{1} & 0 & 0 \\
P_{2} & P_{3} & 0 \\
0 & 0 & \square
\end{array}\right]
$$

with $P_{1}$ is a symmetric and positive-definite matrix.

The difference $\Delta V\left(\tilde{\mathbf{x}}_{k}\right)$ is given by

$$
\Delta V\left(\tilde{\mathbf{x}}_{k}\right)=\Delta V_{1}\left(\tilde{\mathbf{x}}_{k}\right)+\Delta V_{2}\left(\tilde{\mathbf{x}}_{k}\right)+\Delta V_{3}\left(\tilde{\mathbf{x}}_{k}\right)
$$

Let us now compute $\Delta V_{1}\left(\mathbf{x}_{k}\right)$ :

$$
\begin{aligned}
\Delta V_{1}\left(\tilde{\mathbf{x}}_{k}\right) & =V_{1}\left(\tilde{\mathbf{x}}_{k+1}\right)-V_{1}\left(\tilde{\mathbf{x}}_{k}\right)=\tilde{x}_{k+1}^{\top} E^{\top} P \tilde{x}_{k+1}-\tilde{x}_{k}^{\top} E^{\top} P \tilde{x}_{k} \\
& =y_{k}^{\top} P_{1} y_{k}-x_{k}^{\top} P_{1} x_{k}=y_{k}^{\top} P_{1} y_{k}-2\left[\begin{array}{lll}
x_{k}^{\top} & 0 & 0
\end{array}\right] P_{1}\left[\begin{array}{c}
\frac{1}{2} x_{k} \\
0 \\
0
\end{array}\right]
\end{aligned}
$$

which can be rewritten using the fact that $0=-y_{k}+A x_{k}+A_{d} x_{k-d_{k}}$ as follows:

$$
\Delta V_{1}\left(\widetilde{\mathbf{x}}_{k}\right)=\tilde{\mathbf{x}}_{k}^{\top}\left[\left[\begin{array}{ccc}
0 & 0 & 0 \\
0 & P_{1} & 0 \\
0 & 0 & 0
\end{array}\right]-P^{\top}\left[\begin{array}{ccc}
\frac{1}{2} & 0 & 0 \\
A & -\square & A_{d} \\
0 & 0 & 0
\end{array}\right]-\left[\begin{array}{ccc}
\frac{1}{2} \square & A^{\top} & 0 \\
0 & -\square & 0 \\
0 & A_{d}^{\top} & 0
\end{array}\right] P\right] \widetilde{\mathbf{x}}_{k} .
$$

For $V_{2}\left(\widetilde{\mathbf{x}}_{k}\right)$, by standard manipulation, we have

$$
\Delta V_{2}\left(\tilde{\mathbf{x}}_{k}\right)=V_{2}\left(\tilde{\mathbf{x}}_{k+1}\right)-V_{2}\left(\tilde{\mathbf{x}}_{k}\right)=\sum_{l=k+1-d_{k+1}}^{k} x_{l}^{\top} Q x_{l}-\sum_{l=k-d_{k}}^{k-1} x_{l}^{\top} Q x_{l} .
$$

Notice that

$$
\begin{gathered}
\sum_{l=k+1-d_{k+1}}^{k} x_{l}^{\top} Q x_{l}=\sum_{l=k+1-d_{k+1}}^{k-\underline{d}} x_{l}^{\top} Q x_{l}+\sum_{l=k+1-\underline{d}}^{k-1} x_{l}^{\top} Q x_{l}+x_{k}^{\top} Q x_{k}, \\
\sum_{l=k-d_{k}}^{k-1} x_{l}^{\top} Q x_{l}=\sum_{l=k+1-d_{k}}^{k-1} x_{l}^{\top} Q x_{l}+x_{k-d_{k}}^{\top} Q x_{k-d_{k} .}
\end{gathered}
$$


Using this, we can rewrite $\Delta V_{2}\left(\mathbf{x}_{k}\right)$ as follows:

$$
\Delta V_{2}\left(\tilde{\mathbf{x}}_{k}\right)=x_{k}^{\top} Q x_{k}-x_{k-d_{k}}^{\top} Q x_{k-d_{k}}+\sum_{l=k+1-d_{k+1}}^{k-\underline{d}} x_{l}^{\top} Q x_{l}+\sum_{l=k+1-\underline{d}}^{k-1} x_{l}^{\top} Q x_{l}-\sum_{l=k+1-d_{k}}^{k-1} x_{l}^{\top} Q x_{l} .
$$

For $V_{3}\left(\widetilde{\mathbf{x}}_{k}\right)$, one has

$$
\begin{aligned}
\Delta V_{3}\left(\tilde{\mathbf{x}}_{k}\right) & =\sum_{l=-\bar{d}+2}^{-\underline{d}+1} \sum_{m=k+l}^{k} x_{m}^{\top} R x_{m}-\sum_{l=-\bar{d}+2}^{-\underline{d}+1} \sum_{m=k+l-1}^{k-1} x_{m}^{\top} R x_{m} \\
& =\sum_{l=-\bar{d}+2}^{-\underline{d}+1}\left[\sum_{m=k+l}^{k-1} x_{m}^{\top} R x_{m}+x_{k}^{\top} R x_{k}-\sum_{m=k+l}^{k-1} x_{m}^{\top} R x_{m}-x_{k+l-1}^{\top} R x_{k+l-1}\right] \\
& =\sum_{l=-\bar{d}+2}^{-\underline{d}+1}\left[x_{k}^{\top} R x_{k}-x_{k+l-1}^{\top} R x_{k+l-1}\right]=(\bar{d}-\underline{d}) x_{k}^{\top} R x_{k}-\sum_{l=-\bar{d}+2}^{-\underline{d}+1} x_{k+l-1}^{\top} R x_{k+l-1} \\
& =(\bar{d}-\underline{d}) x_{k}^{\top} R x_{k}-\sum_{l=k+1-\bar{d}}^{k-\underline{d}} x_{l}^{\top} R x_{l} .
\end{aligned}
$$

Notice that $d_{k} \geq \underline{d}$ for all $k$, we get

$$
\begin{gathered}
\sum_{l=k+1-\underline{d}}^{k-1} x_{l}^{\top} Q x_{l} \leq \sum_{l=k+1-d_{k}}^{k-1} x_{l}^{\top} Q x_{l}, \quad \sum_{l=k+1-d_{k+1}}^{k-\underline{d}} x_{l}^{\top} Q x_{l} \leq \sum_{l=k+1-\bar{d}}^{k-\underline{d}} x_{l}^{\top} Q x_{l}, \\
\sum_{l=k+1-\bar{d}}^{k-\underline{d}} x_{l}^{\top} Q x_{l}<\sum_{l=k+1-\bar{d}}^{k-\underline{d}} x_{l}^{\top} R x_{l}, \quad \text { since } Q<R .
\end{gathered}
$$

Finally, by using (3.9), (3.12), and (3.13) together with these inequalities and the condition (3.2), we obtain

$$
\Delta V\left(\tilde{\mathbf{x}}_{k}\right) \leq\left[\begin{array}{lll}
x_{k}^{\top} & y_{k}^{\top} & x_{k-d_{k}}^{\top}
\end{array}\right] M\left[\begin{array}{c}
x_{k} \\
y_{k} \\
x_{k-d_{k}}
\end{array}\right]<0
$$

where

$$
M=\left[\begin{array}{ccc}
Q+(\bar{d}-\underline{d}) R-P_{1}-A^{\top} P_{2}-P_{2}^{\top} A & -A^{\top} P_{3}+P_{2}^{\top} & -P_{2}^{\top} A_{d} \\
-P_{3}^{\top} A+P_{2} & P_{1}+P_{3}+P_{3}^{\top} & -P_{3}^{\top} A_{d} \\
-A_{d}^{\top} P_{2} & -A_{d}^{\top} P_{3} & -Q
\end{array}\right] .
$$

This implies that the system is stable.

Remark 3.2. The conditions of this theorem are delay dependent and can solve many stability problems for discrete-time systems with time-varying delay. These conditions are more appropriate for practical systems since practically it is impossible to know exactly the delay but lower and upper bounds are always possible. 
Remark 3.3. In practice we are always interested in knowing what would be the maximum time delay such that the system can remain stable. The answer to this question can be obtained by solving the following nonlinear optimization problem:

$$
\max _{\bar{d}>0, P_{1}>0, P_{2}, P_{3}, Q>0, R>0} \bar{d} \quad \text { s.t }(3.1)-(3.2) .
$$

When time delay is constant, that is, $d_{k}=d$ and $d=\bar{d}=\underline{d}$, the results given by Theorem 3.1 are replaced by the following ones.

Corollary 3.4. If there exist symmetric and positive-definite matrices $P_{1}>0, Q>0$, and matrices $P_{2}$ and $P_{3}$ such that the following LMI holds:

$$
\left[\begin{array}{ccc}
Q-P_{1}-A^{\top} P_{2}-P_{2}^{\top} A & -A^{\top} P_{3}+P_{2}^{\top} & -P_{2}^{\top} A_{d} \\
-P_{3}^{\top} A+P_{2} & P_{1}+P_{3}+P_{3}^{\top} & -P_{3}^{\top} A_{d} \\
-A_{d}^{\top} P_{2} & -A_{d}^{\top} P_{3} & -Q
\end{array}\right]<0,
$$

then system (2.1) is stable.

Remark 3.5. The conditions of this corollary are delay independent, which makes them conservative.

\section{Stabilizability}

The aim of this section is to design a state-feedback controller which stabilizes the resulting closed-loop system. Using the controller expression (2.3) and system dynamics (2.1), we get the following dynamics for the closed-loop system:

$$
x_{k+1}=[A+B K] x_{k}+A_{d} x_{k-d_{k}} .
$$

If we let $\bar{A}=A+B K$, based on the results on stability, the closed-loop system will be stable if there exist symmetric and positive-definite matrices $P_{1}>0, Q>0, R>0$, and matrices $P_{2}$ and $P_{3}$ that satisfy the following LMIs:

$$
\begin{gathered}
Q<R, \\
{\left[\begin{array}{ccc}
Q+(\bar{d}-\underline{d}) R-P_{1}-\bar{A}^{\top} P_{2}-P_{2}^{\top} \bar{A} & -\bar{A}^{\top} P_{3}+P_{2}^{\top} & -P_{2}^{\top} A_{d} \\
-P_{3}^{\top} \bar{A}+P_{2} & P_{1}+P_{3}+P_{3}^{\top} & -P_{3}^{\top} A_{d} \\
-A_{d}^{\top} P_{2} & -A_{d}^{\top} P_{3} & -Q
\end{array}\right]<0 .}
\end{gathered}
$$

If we denote by $X$ the inverse of $P$, we have

$$
X=\left[\begin{array}{ccc}
X_{1} & 0 & 0 \\
X_{2} & X_{3} & 0 \\
0 & 0 & \square
\end{array}\right], \quad X_{1}=P_{1}^{-1}, \quad 0=P_{2} X_{1}+P_{3} X_{2}, \quad X_{3}=P_{3}^{-1}
$$


Pre- and postmultiplying the second LMI, respectively, by $X^{\top}$ and $X$ and using these relations, we get

$$
\left[\begin{array}{ccc}
X_{1}^{\top} Q X_{1}+(\bar{d}-\underline{d}) X_{1}^{\top} R X_{1}-X_{1}+X_{2}^{\top} P_{1} X_{2} & \star & \star \\
-A X_{1}-B K X_{1}+X_{2}+X_{3}^{\top} P_{1} X_{2} & X_{3}^{\top} P_{1} X_{3}+X_{3}+X_{3}^{\top} & \star \\
0 & -A_{d}^{\top} & -Q
\end{array}\right]<0
$$

which can be rewritten as after letting $Y=K X_{1}$ :

$$
\begin{aligned}
& {\left[\begin{array}{cc}
X_{1}^{\top} Q X_{1}+(\bar{d}-\underline{d}) X_{1}^{\top} R X_{1}-X_{1} & \star \\
-A X_{1}-B Y+X_{2} & X_{3}+X_{3}^{\top}
\end{array}\right]+\left[\begin{array}{c}
X_{2}^{\top} \\
X_{3}^{\top}
\end{array}\right] P_{1}\left[\begin{array}{ll}
X_{2} & X_{3}
\end{array}\right]} \\
& +\left[\begin{array}{c}
0 \\
-A_{d} Q^{-1}
\end{array}\right] Q\left[\begin{array}{ll}
0 & -Q^{-1} A_{d}^{\top}
\end{array}\right]<0,
\end{aligned}
$$

which gives in turn

$$
\left[\begin{array}{cccccc}
-X_{1} & \star & \star & \star & \star & \star \\
-A X_{1}-B Y+X_{2} & X_{3}+X_{3}^{\top} & \star & \star & \star & \\
X_{2} & X_{3} & -X_{1} & \star & \star & \\
0 & -Q^{-1} A_{d}^{\top} & 0 & -Q^{-1} & \star & \star \\
X_{1} & 0 & 0 & 0 & -Q^{-1} & \star \\
X_{1} & 0 & 0 & 0 & 0 & -\frac{1}{\bar{d}-\underline{d}} R^{-1}
\end{array}\right]<0 .
$$

Letting $S=Q^{-1}$ and $T=R^{-1}$, we get the following results.

Theorem 4.1. For a given set of upper and lower bounds on the time-varying delay $d_{k}, \bar{d}$, and $\underline{d}$, if there exist symmetric and positive-definite matrices $X_{1}>0, S>0$, and $T>0$ and matrices $X_{2}>0$ and $X_{3}>0$ such that the following LMIs hold:

$$
\begin{aligned}
& T<S \text {, } \\
& {\left[\begin{array}{cccccc}
-X_{1} & \star & \star & \star & \star & \star \\
-A X_{1}-B Y+X_{2} & X_{3}+X_{3}^{\top} & \star & \star & \star & \star \\
X_{2} & X_{3} & -X_{1} & \star & \star & \star \\
0 & -S A_{d}^{\top} & 0 & -S & \star & \star \\
X_{1} & 0 & 0 & 0 & -S & \star \\
X_{1} & 0 & 0 & 0 & 0 & -\frac{1}{\bar{d}-\underline{d}} T
\end{array}\right]<0,}
\end{aligned}
$$

then system (2.1) is stable under the controller (2.3) and the control gain is given by $K=$ $Y X_{1}^{-1}$.

Similarly, when the time delay is constant, that is, $d_{k}=d, d=\bar{d}=\underline{d}$, Theorem 4.1 is reduced to the following corollary. 
8 Discrete-time systems with time-varying time delay

Corollary 4.2. If there exist symmetric and positive-definite matrices $X_{1}>0$ and $S>0$ and matrices $X_{2}>0$ and $X_{3}>0$ such that the following LMI holds:

$$
\left[\begin{array}{ccccc}
-X_{1} & \star & \star & \star & \star \\
-A X_{1}-B Y+X_{2} & X_{3}+X_{3}^{\top} & \star & \star & \star \\
X_{2} & X_{3} & -X_{1} & \star & \star \\
0 & -S A_{d}^{\top} & 0 & -S & \star \\
X_{1} & 0 & 0 & 0 & -S
\end{array}\right]<0
$$

then system (2.1) is stable under the controller (2.3) and the control gain is given by $K=$ $Y X_{1}^{-1}$.

Remark 4.3. To find the maximal upper bound of the time delay, $\bar{d}$, for which system (2.1) is stable, we need to solve the following optimization problem:

$$
\max _{\bar{d}>0, X_{1}>0, X_{2}, X_{3}, Y, S>0, T>0} \bar{d} \quad \text { s.t }(4.7) .
$$

\section{Numerical examples}

To illustrate the usefulness of the previous theoretical results, let us give the following numerical example.

Example 5.1. Let us consider a system described by (2.1) and suppose that the system data are as follows:

$$
A=\left[\begin{array}{cc}
0 & 1 \\
-2 & -3
\end{array}\right], \quad A_{d}=\left[\begin{array}{cc}
0.01 & 0.1 \\
0.0 & 0.1
\end{array}\right], \quad B=\left[\begin{array}{c}
0.0 \\
1.0
\end{array}\right], \quad \bar{d}=10, \quad \underline{d}=1 .
$$

Solving LMI (4.7), we get

$$
\begin{aligned}
& X_{1}=\left[\begin{array}{ll}
1.0917 & 0.0116 \\
0.0116 & 0.4353
\end{array}\right]>0, \quad X_{2}=\left[\begin{array}{cc}
0.0017 & 0.0673 \\
-0.0000 & -0.0028
\end{array}\right], \\
& X_{3}=\left[\begin{array}{ll}
-0.9270 & -0.0263 \\
-0.0184 & -0.4209
\end{array}\right], \quad S=\left[\begin{array}{cc}
15.3477 & 0.0758 \\
0.0758 & 11.3502
\end{array}\right]>0 \text {, } \\
& T=\left[\begin{array}{cc}
12.7561 & 0.0936 \\
0.0936 & 8.5862
\end{array}\right]>0, \quad Y=\left[\begin{array}{ll}
2.2177 & 1.2879
\end{array}\right] \text {. }
\end{aligned}
$$

Therefore, the controller (2.3) with gain

$$
K=\left[\begin{array}{ll}
2.0005 & 2.9051
\end{array}\right]
$$

stabilizes the system under study.

We have simulated these discrete-time systems with time varying delay with the controller we designed and the results are illustrated in Figure 5.1 and Figure 5.2. These figures show that the closed-loop system is stable under the computed state feedback controller. 


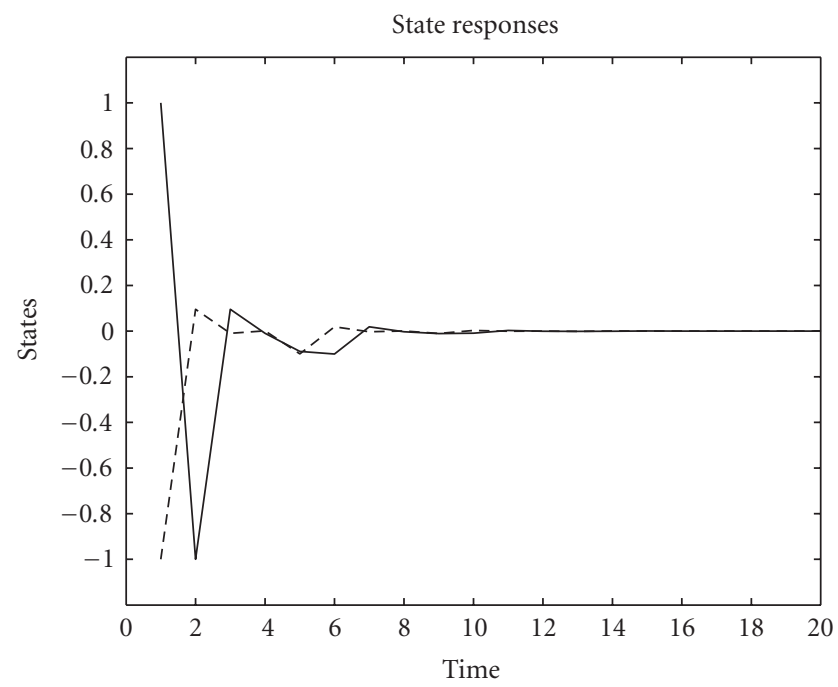

Figure 5.1. The behaviors of the states $x_{1}(t)$ and $x_{2}(t)$ in function of time $t$.

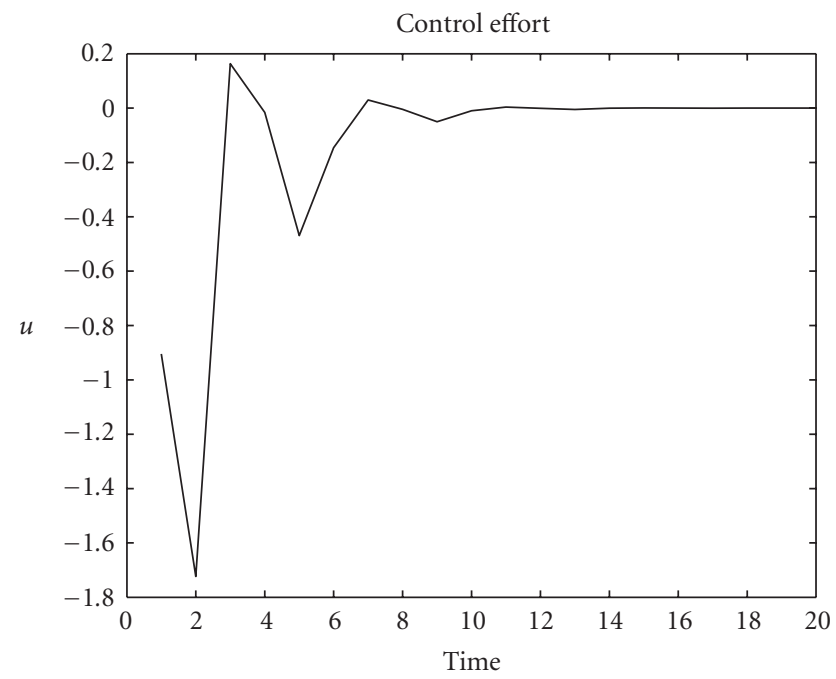

Figure 5.2. The behaviors of the control law $u(t)$ in function of time $t$.

\section{Conclusion}

In this paper, we have studied the problems of stability and stabilizability for a class of linear discrete-time systems with varying time delay in the state. Sufficient conditions in the LMI formalism have been developed to solve the above problems. An algorithm to design the stabilizing state feedback controller is also provided. A Numerical example is included to demonstrate the effectiveness of the proposed techniques. 


\section{References}

[1] E.-K. Boukas and Z. K. Liu, Robust $H_{\infty}$ control of discrete-time Markovian jump linear systems with mode-dependent time-delays, IEEE Transactions on Automatic Control 46 (2001), no. 12, 1918-1924.

[2] —_ Deterministic and Stochastic Time Delay Systems, Birkhäuser, Massachusetts, 2002.

[3] E.-K. Boukas and P. Shi, $\mathscr{H}_{\infty}$-control for discrete-time linear systems with Markovian jumping parameters, Proceedings of 36th IEEE Conference on Decision and Control, California, 1997, pp. 4134-4139.

[4] Y.-C. Chang, S.-F. Su, and S.-S. Chen, LMI approach to static output feedback simultaneous stabilization of discrete-time interval systems with time delay, Proceedings of International Conference on Machine Learning and Cybernetics, vol. 7, Shanghai, 2004, pp. 4144-4149.

[5] H. Gao, J. Lam, C. Wang, and Y. Wang, Delay-dependent output-feedback stabilisation of discretetime systems with time-varying state delay, IEE Proceedings-Control Theory and Applications 151 (2004), no. 6, 691-698.

[6] J. H. Kim and H. B. Park, $H^{\infty}$ state feedback control for generalized continuous/discrete time-delay system, Automatica 35 (1999), no. 8, 1443-1451.

[7] H. Mukaidani, S. Sakaguchi, and T. Tsuji, LMI-based neurocontroller for guaranteed cost control of uncertain time-delay systems, Proceedings of IEEE International Symposium on Circuits and Systems, vol. 4, Kobe, 2005, pp. 3047-3050.

[8] P. Shi, E.-K. Boukas, and R. K. Agarwal, Control of Markovian jump discrete-time systems with norm bounded uncertainty and unknown delay, IEEE Transactions on Automatic Control 44 (1999), no. 11, 2139-2144.

[9] S.-H. Song and J.-K. Kim, $H_{\infty}$ control of discrete-time linear systems with norm-bounded uncertainties and time delay in state, Automatica 34 (1998), no. 1, 137-139.

El-Kébir Boukas: Département de Génie Mécanique, École Polytechnique de Montréal, C.P. 6079, succ. Centre-Ville, Montréal, QC, Canada H3C 3A7

E-mail address: el-kebir.boukas@polymtl.ca 


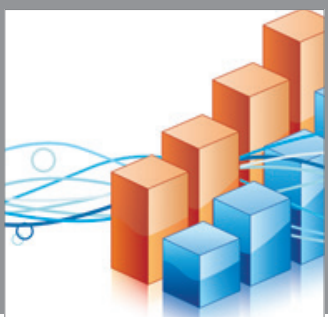

Advances in

Operations Research

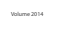

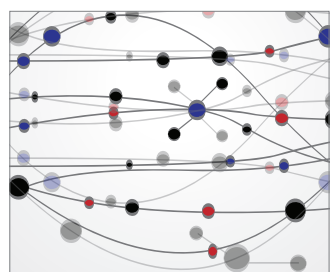

\section{The Scientific} World Journal
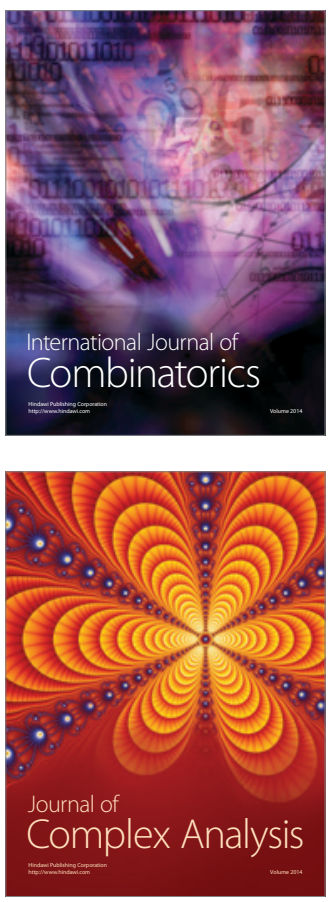

International Journal of

Mathematics and

Mathematical

Sciences
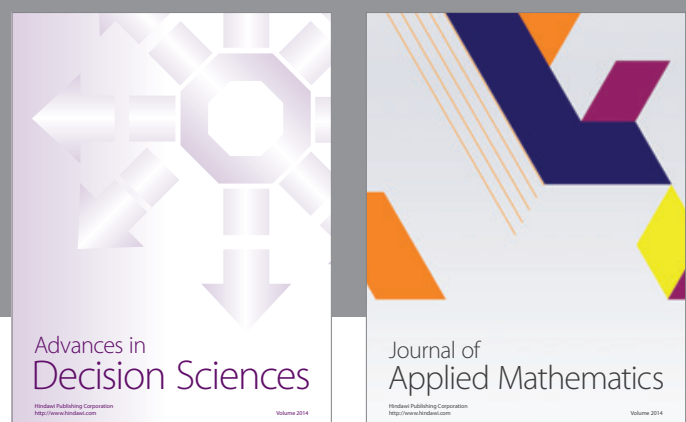

Journal of

Applied Mathematics
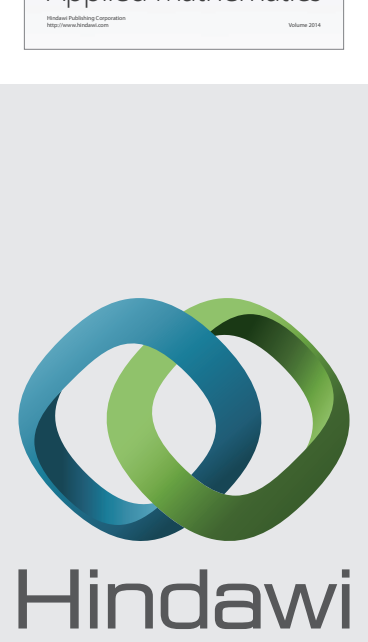

Submit your manuscripts at http://www.hindawi.com
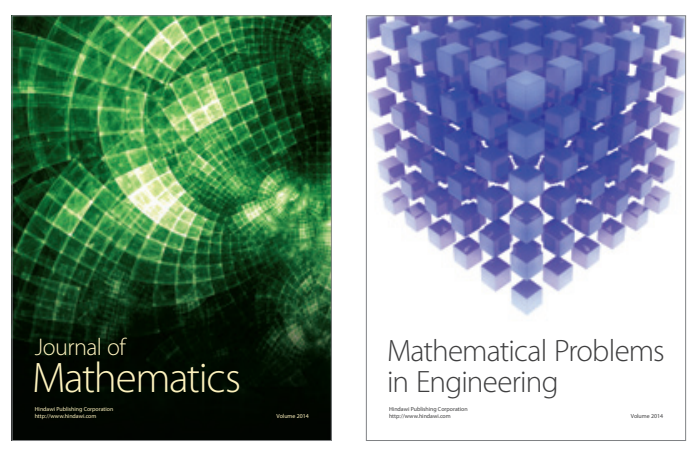

Mathematical Problems in Engineering
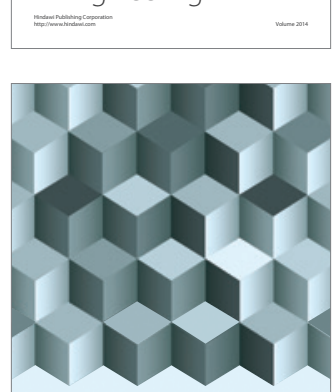

Journal of

Function Spaces
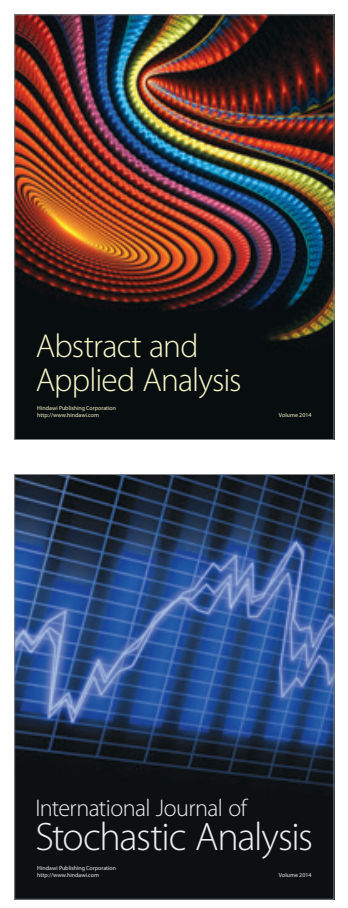

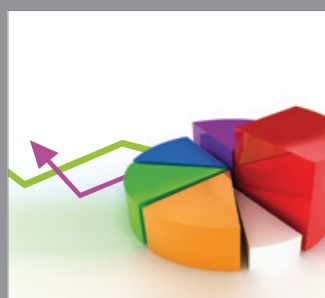

ournal of

Probability and Statistics

Promensencen
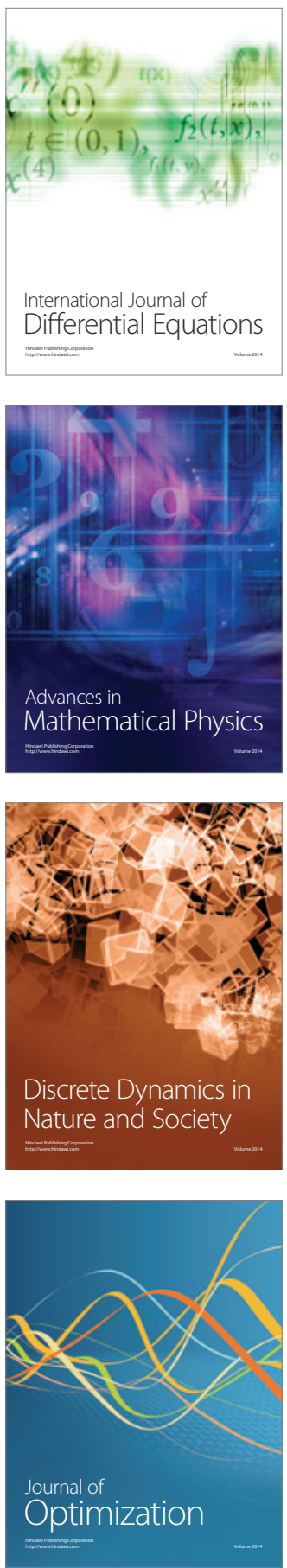